\title{
A PROTOTYPE OF FEATURE-BASED DESIGN FOR ASSEMBLY
}

\author{
by (listed alphabetically) \\ T. L. De Fazio \\ A. C. Edsall \\ R. E. Gustavson \\ J. A. Hernandez \\ P. M. Hutchins \\ H.W. Leung \\ S. C. Luby \\ R.W. Metzinger \\ J.L. Nevins \\ K. K. Tung \\ D. E. Whitney
}

The Charles Stark Draper Laboratory, Inc.

Cambridge, MA 02139

Work supported through DARPA Contract MDA972-88-C-0027

\begin{abstract}
This paper describes a prototype software system that implements a form of featurebased design for assembly. It is not an automated design system but instead a decision and design aid for designers interested in Concurrent Design. Feature-based design captures design intent (assembly topology, product function, manufacturing, or field use) while creating part and product geometry. Design for assembly as used here extends existing ideas about critiquing part shapes and part count to include assembly process planning, assembly sequence generation, assembly fixturing assessments, and assembly process costs. This work was primarily interested in identifying the information important to DFA tasks, and how that information could be captured using feature-based design. It was not intended to extend the state of the art in feature-based geometry creation, but rather to explore the uses of the information that can be captured. The prototype system has been programmed in LISP on Sun workstations. Its research contributions comprise integration of feature-based design with several existing and new assembly analysis and synthesis algorithms; construction of feature properties to meet the needs of those algorithms; a carefully chosen division of labor between designer and computer, and illustration of feature-based models of products as the information source for assembly analysis and process design. Some of its functions have been implemented approximately or partially but they give the flavor of the benefits to be expected from a fully functional system.
\end{abstract}




\section{INTRODUCTION}

Need

There is a general need for better quality designs of manufactured products and their parts. It is widely believed that $70 \%$ or more of the life cycle cost of a product is determined during the early design process and there is intense interest in developing design tools and aids that will make design more effective. At present, however, most computer tools for aiding design are directed at creation of geometry or pictures of geometry, together with some analytical capability for functional performance such as finite element analysis. Even these tools do not provide any guidance or advice to help improve the design. Little has been done to link geometric models to cost analyses, process planning for fabrication or assembly, tolerance analysis, quality analysis or other aspects of product design and manufacture. Furthermore, there are few or no computerized tools to aid these analyses.

A major consequence of the lack of tools is that those who wish to improve design by using Concurrent Design [2] must do so almost totally manually. There is no comprehensive computer-aided design environment with design for manufacturing and assembly tools that will give a designer rapid feedback concerning cost, producibility, or other important criteria. Lacking this, companies expand the design team with experts from other domains who also lack even specialized computer aided tools. Their input undoubtedly improves the design but large teams can be cumbersome, possibly defeating some of the purpose of forming them. Furthermore, since existing computer design aids focus on functional design rather than fabrication or assembly, the latter constituencies lack ammunition and credibility in the design negotiating process.

\section{Desirable Properties of a Design for Manufacturing and Assembly (DFMA) System}

What would a useful DFMA system be like and how would we create one? Ideally, it would be a workstation-like environment at which a designer could create a design in terms beyond just geometry, and have access to capabilities for design tradeoff studies, cost reduction studies, cost driver identification, producibility evaluations, design rule checking, and manufacturing and assembly evaluations and recommendations. That is, it would act like a manufacturing expert looking over the designer's shoulder, providing suggestions, comments, and information about fabrication and assembly.

To support such an environment, we must create a method of describing designs, one that can absorb the information necessary to carry out the relevant analyses. This information must be in the designer's customary vocabulary. Most important, the environment must provide an incentive for the designer to use it, such as permitting creation of designs that will stand scrutiny on DFMA and cost criteria. Our approach to this challenge is to begin with feature-based design.

\section{Definition of Feature-Based Design (FBD) and Feature-Based Design for Assembly (FBDA)}

Feature-based design is a technique that permits a designer to express design intent while creating the geometry of the product.[1] It both requires and permits the designer to 
think beyond mere shape and to state explicitly what portions of a part are important and why.

A "feature" is any geometric or non-geometric attribute of a discrete part whose presence or dimensions are relevant to the product's or part's function, manufacture, engineering analysis, use, etc., or whose availability as a primitive or operation facilitates the design process. Tables 1 and 2 give some examples.

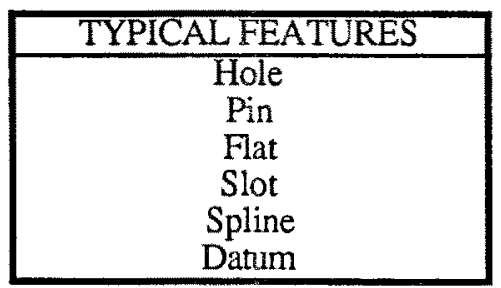

Table 1. Examples of Features

\begin{tabular}{|c|}
\hline TYPICAL FEATURE ATTRIBUTES \\
\hline Diameter \\
Depth \\
Tolerance \\
Orientation (WRT part coordinates) \\
Used for (mating, fixturing, test,...) \\
Mates-with (feature \# $\mathrm{xx}$ on part \# yy) \\
\hline
\end{tabular}

Table 2. Examples of Feature Attributes

Recently, considerable work has been done in feature-based design, but it focused mostly on ease of part geometry creation and information for evaluating individual part fabrication, especially machining. [3-7] Since our focus is assembly, the rest of this paper describes feature-based DFA but it should be remembered that the larger challenge is to include in a feature-based approach all the other aspects of design and manufacture as well. We are developing a structure that will be expandable.

We will show that much of the information needed for DFA can be captured during the actual creation of the design geometry, through an extended feature-based modeling system. Once the design has been described in terms of features, the computer can analyze it using a variety of techniques, including heuristics, directed search, geometric reasoning, numerical analyses, and so on. These techniques gain much of their power from the designer's geometric and non-geometric information contained in the features of the parts and assemblies.

\section{Intellectual Challenges} include:

Implementing feature-based design poses several intellectual challenges. These

- what is a part that is designed using features? 
This is not a trivial issue, since with currently available tools it is too tedious to design every region of a part using features. It would also defeat an important purpose of features, namely to focus attention to those few areas that need it. For these reasons, we define (for now) a featurized part as any geometric shape onto which features have been attached or portions of which have been singled out as features. This definition may be supplanted as the field matures.

- what information is really needed to support FBDFA?

We have identified two classes of data. These are part data and assembly data. Part data include all the usual functional data (geometry, mass distribution, material, dimensioning scheme) plus tolerances, feature existence, feature attributes, and feature use. Assembly data include inter-part dimensions, tolerances, clearances, feature mate type, presence of lubricants or adhesives, degrees of freedom in the mate, permission to grip or fixture on the feature, and others yet to be identified. We use this information in generating and evaluating assembly sequences, process plans, and cost estimates.

- how should feature-level information be entered?

Here there seem to be three possibilities. The computer could recognize the features; the designer could point out what collections of existing geometry constitute features; or the designer could call them forth parametrically from a library. The latter two seem to be the most likely to be useful in the long run since recognition appears to be limited to geometric information and the designer would have to add the other information anyway.

- how to deal with features at the appropriate time?

Designers are used to making a layout drawing first and then adding details to individual parts. The input is sketchy at first and becomes elaborated later. Feature-based design needs to be able to support a range of design styles; among these could be rough geometry followed by feature specifications, or feature sketches (using icons?) right from the beginning.

- how to deal with parts having "layers of features"?

"Layers of features" means having features that are obliterated during processing and replaced by other features. Forging followed by machining is an example. We raise this and the ones that follow without making specific suggestions at this time.

- how to define features that are subsets of or overlap other features?

An example is a keyway in a shaft end. Another is identification of the cylindrical part of a shaft end as a bearing-mate feature and identification of both the cylindrical part plus the flat end as a fixture-mate feature.

- what other feature-based design information is required for operation of the business, such as purchasing or production management? 


\section{Benefits}

In addition to the benefits cited above for better design tools in general, FBD and FBDFA offer specific benefits. These include allowing the designer to

- operate in a high level vocabulary ("Create a countersunk 1/4-20 NC threaded hole here.")

- orient parts with respect to each other easily by referring to the features that mate (and automatically create the mating database referred to below)

- maintain dependencies between features such as correct diameters for pin and hole that will be press fit

- check adherence to design rules, such as ensuring that at least pin or bore has a chamfer or that bolt torques are appropriate to their size and material

- discover difficult mating situations based on feature attributes, materials, or tolerances

\section{CAPABILITIES OF CURRENT PROTOTYPE}

\section{Implementation}

A prototype software system has been written that embodies several of the above properties. It runs on Sun 3 workstations and employs LISP, C, and I-DEAS ${ }^{\mathrm{TM}}$ (SDRC, Inc.). A summary description follows, and subsequent sections give more detail. References $2,6,8-11,13$, and 14 describe the underlying theory of assembly sequence analysis and editing as well as assembly process planning, economic analysis, and assembly system synthesis. These references also contain further references to the general literature in these areas.

The particular contributions of this paper and the prototype it describes are

- integration of capabilities into an end-to-end design system not achieved before to our knowledge

- creation of assembly feature requirements based on prior research on algorithms (for assembly sequence analysis, process planning, system design, etc.) that call for information that properly designed features can provide

- observance of some distinctions between what a person can do (such as geometric analysis or judgement of assembly difficulty) and what a computer can do (sort through thousands of alternatives, create graphs, perform arithmetic, etc) with the result that the prototype described here combines a high level of credibility in its output with fairly fast response at the cost (benefit, really) of involving the human designer in the process.

The work described here was not intended to extend the state of the art in featurebased modeling but rather to show the potential for feature-based modeling in assembly process design. Most of the algorithms included are well-tested on real products and were developed based on our experience using concurrent design in industry. 


\section{Summary Capabilities}

Figure 1 shows in schematic form the topics in a typical design of a product with discrete parts, after the functional requirements have been established, and highlights the capabilities and potential for feature-based design for assembly. ${ }^{1}$

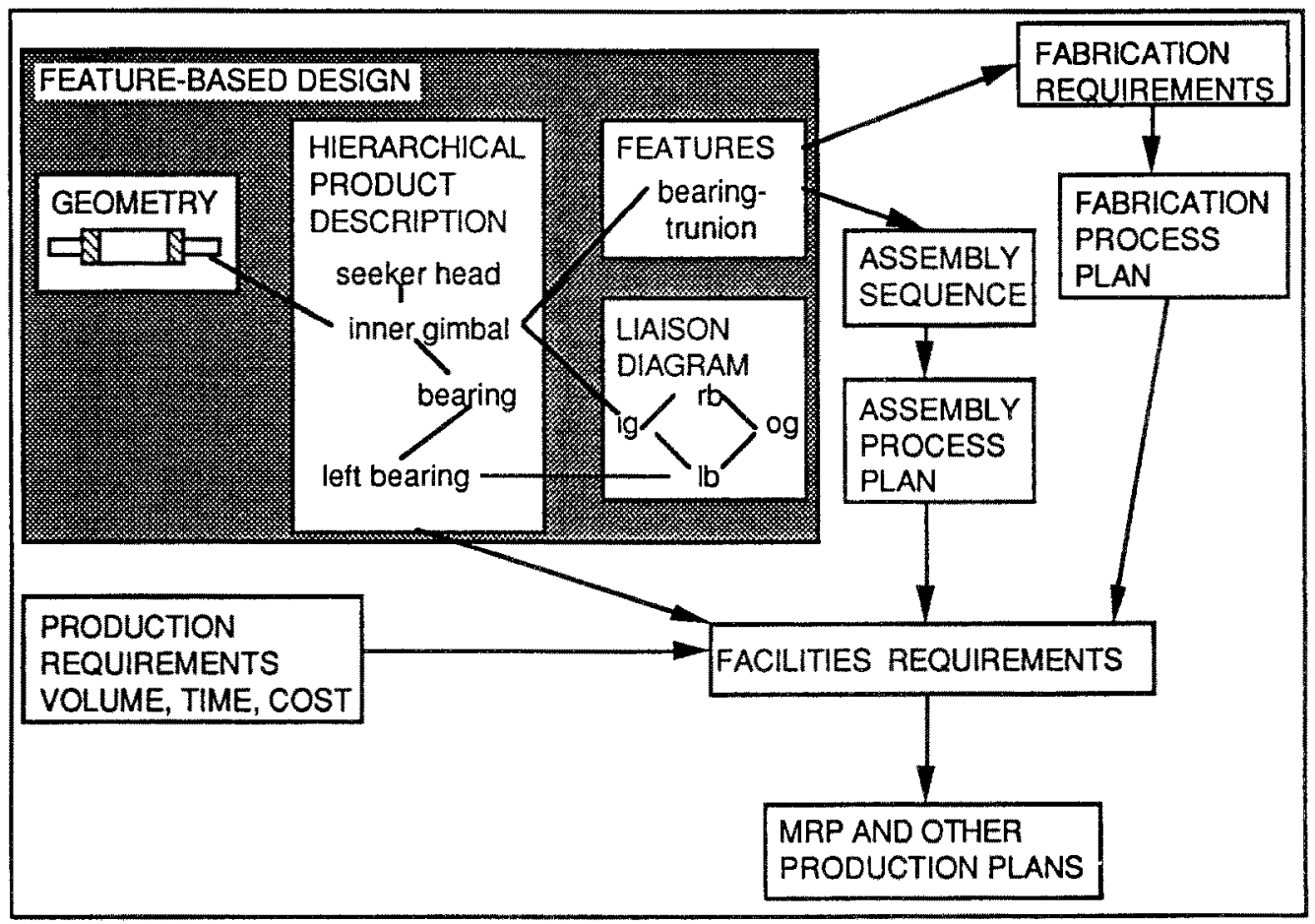

Figure 1. Relation of Feature-based Design to the Process of Designing a Discrete Parts Product. The prototype software described in this paper includes all the entities in this figure except those dealing with fabrication and MRP.

Figure 2, shows in schematic form the design process as supported by the current prototype software. Upon starting the software, the user constructs a feature-level solid model of the product, then assembles the parts by indicating feature mates. Features and assembly information are captured in a database in hierarchical form with all the parts and their features, plus mating data in the form of a liaison diagram [8]. ${ }^{2}$

1 of course, there may be revisions to the requirements as design proceeds. These aspects of concurrent design are not discussed here.

${ }^{2} \mathrm{~A}$ liaison represents a mate between two parts. 


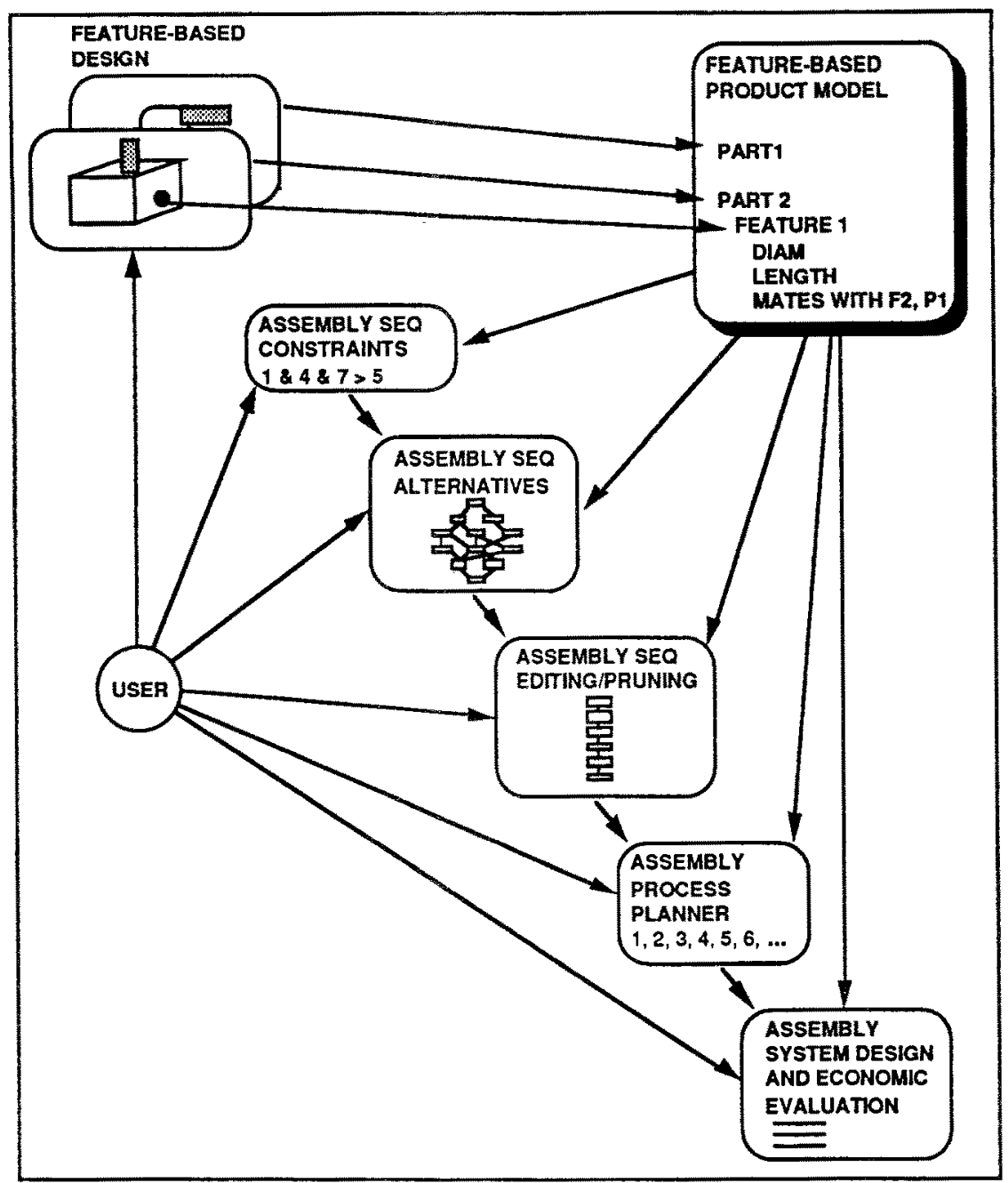

Figure 2. Sketch of the Design Process Supported by the Software

Next, the user invokes the assembly sequence generator, which uses a questionanswer dialog [8] with the designer plus the geometric features to determine the legal assembly operations and generate all the feasible assembly sequences. The designer may then edit the sequences by optionally eliminating assembly states, assembly actions, assembly plans with parallel operations or with converging assembly lines, plus other explicit restrictions, until a satisfactory set of sequences is obtained.

The designer transfers these sequences to an assembly process planner which uses feature information and user input to plan assembly. The assembly plan contains information on part or subassembly orientation, size, weight, and task type. This 
information is passed to an assembly system design module [9] which musters assembly resources and creates a least cost assembly system for each candidate sequence which will meet the required production rate and investment targets. The designer may return to any place in this process and make revisions to the original design, select a different assembly sequence, change cost or production requirements, and repeat the process.

Each of the above steps is described in more detail below.

\section{Eeature-Based Parts and Assembly Modeler}

We have developed a features-level parts and assembly modeler on top of SDRC's IDEASTM package for the Sun using SDRC's "IDEAL" programming language and PEARL ${ }^{\mathrm{TM}}$ relational database facility. This feature-based modeling layer is more than a set of "macro's," which would simply multiply a user's power to generate geometry using higher level commands. Instead, it builds a database of feature-level information as the user works on a design. At the conclusion of a design session, this database is passed along to the downstream analysis applications (assembly process analysis and economic evaluations), affording them access at this higher features level to the designer's thinking about the design.

As is the case in the underlying I-DEAS, our feature-level modeler is divided into two main modes: Part Modeling and Assembly Modeling.

In Part Modeling, the designer can directly create Pin, Bore, and Slot features on a part. In our current implementation, the first step for each part is to create an initial piece of geometry which serves as the base to be augmented or modified by subsequent feature modeling. In addition, we provide some capabilities for the designer to point out features that are represented by existing geometry. This capability allows for cases where it is today simpler to create some geometry using primitive solids (e.g., by Revolving a profile) as well as to allow for feature types (e.g. Flats) that are not easily made up from primitive solids. Information typically requested from the user or inferred from his/her mouse actions are shown in Table 3. All non-geometric information is stored as text in PEARL.

\begin{tabular}{|c|c|c|}
\hline & Provided by User & Computed \\
\hline $\begin{array}{l}\text { For Parts and } \\
\text { Assemblies }\end{array}$ & $\begin{array}{l}\text { name, density, shape, } \\
\text { size }\end{array}$ & $\begin{array}{l}\text { weight, CG, inertia } \\
\text { properties, bounding } \\
\text { box (for process } \\
\text { planning) }\end{array}$ \\
\hline For Features & $\begin{array}{l}\text { ID, description, } \\
\text { purpose, shape, size, } \\
\text { location on part }\end{array}$ & $\begin{array}{l}\text { reference direction (for } \\
\text { assembly analysis and } \\
\text { process planning) }\end{array}$ \\
\hline
\end{tabular}

Table 3. Data About Features

Other information could be requested, such as tolerances, surface finishes, thread sizes, and so on, and used for further assembly modeling or by other software modules not included now, such as for fabrication planning. 
Duplicate parts may be defined first as separate entities (e.g., 3/8" bearing), and copies (e.g., left $3 / 8$ " bearing, right $3 / 8$ " bearing) may be made as needed and included in the product. Each feature is an instance of existing feature library entries but at present the user cannot create new entries.

Figures 3 and 4 show a product comprising 18 parts that was constructed in this way. It is the mechanical structure of a generic seeker head, a component of tactical air-air missiles.

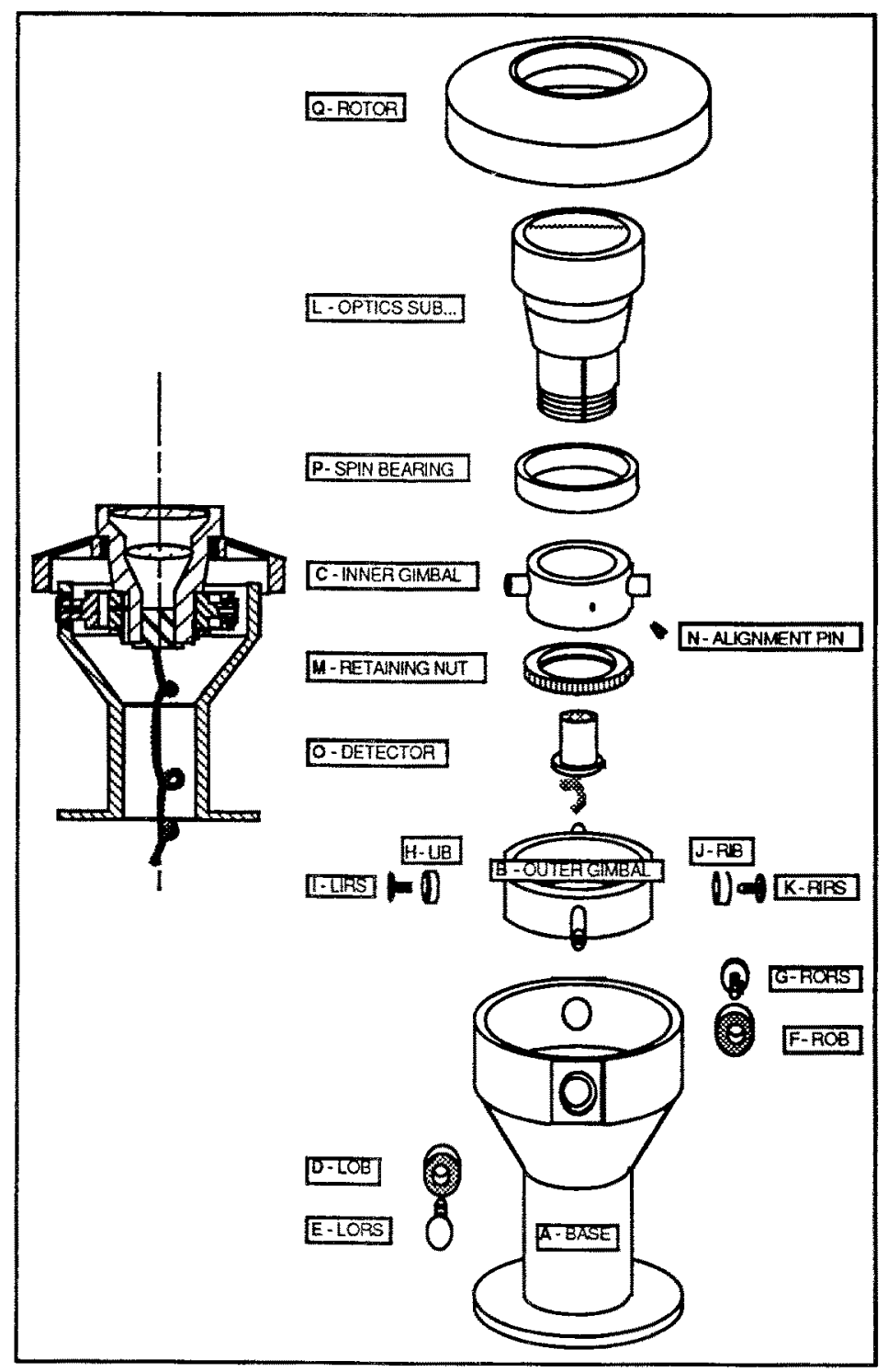

Figure 3. Generic Seeker Head Product 


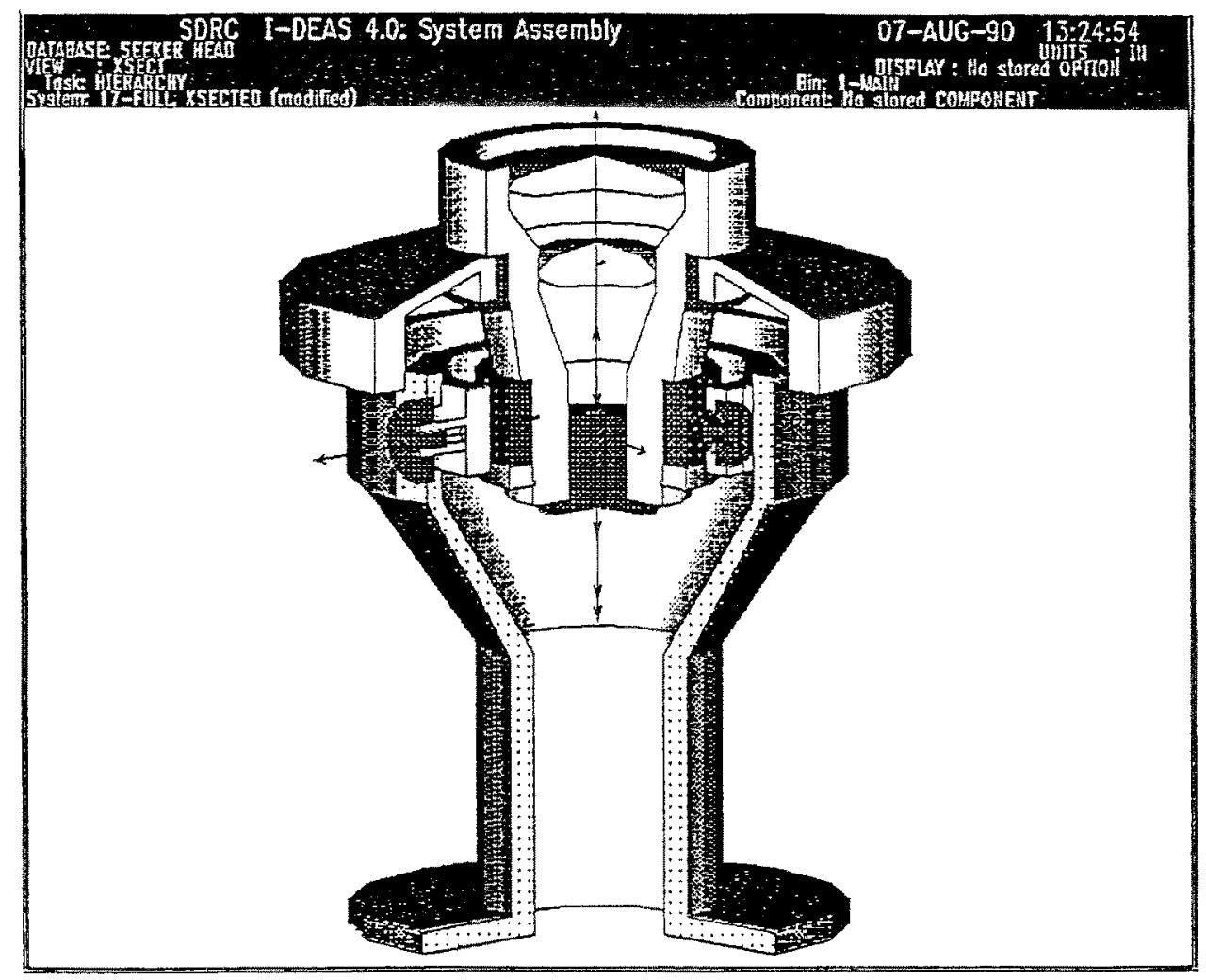

Figure 4. Sectioned View of the Seeker Head Geometric Model Showing Parts with Some Feature Codenames Visible 
When the designer is finished making all the parts, he/she may switch to the assembly modeler and model the final assembly state of the product. This is done by calling forth the parts and typing the names of the features that mate them. The software uses information in the database concerning the reference directions and locations of the features to align the parts, a technique that yields the correct positions most of the time. This method is a considerable improvement over the corresponding facility provided by I-DEAS ${ }^{\mathrm{TM}}$, which requires the user to work at the geometry level with cumbersome orientation commands. Figure 5 shows this action occurring for the base and outer gimbal of the seeker head.

Our implementation of assembly modeling via features is intended to exemplify the improvement in user-computer interaction afforded by raising the discourse to the features level. It is not complete and currently cannot correctly mate every kind of feature we can define, but there is no barrier other than programming time to such extensions.

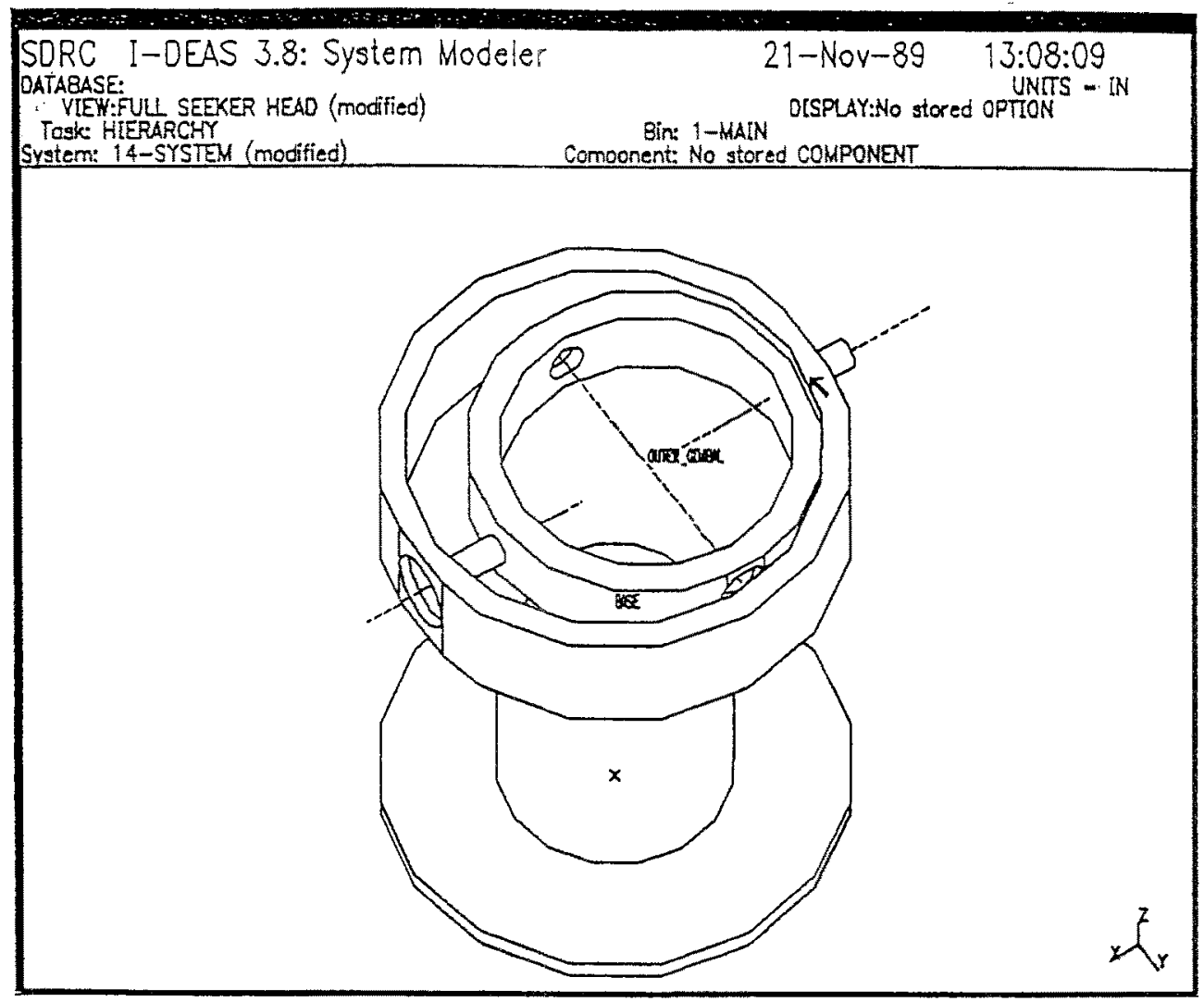

Figure 5. Outer Gimbal Being Oriented with Respect to the Base Via Specification of the Mate Between the Pin on the Gimbal and the Bearing Bore in the Base. 
During feature-based modeling, our program layer builds a database of the higherlevel feature information using the I-DEAS ${ }^{\mathrm{TM}}$ PEARL ${ }^{\mathrm{TM}}$ relational database facility. At completion of modeling, the program writes out the database into a text file which is read by LISP code and converted into an object database that contains all the logical and textual information created during the I-DEAS ${ }^{\mathrm{TM}}$ session. This information includes the logical structure of the product, such as what the parts are, what mates to what and via what features, and what type of feature mate is involved. Information that can be inferred includes clearances and degrees of freedom of mates, relative extraction directions for mating features, total weight and bounding box size of the product or any subassembly, distance between two features on different parts and (in future implementations) tolerances between such features. Also possible in the future is interpretation of assembly difficulty using Boothroyd [11] or Draper [2] methods.

\section{Assembly Sequence Constraints}

Before the assembly sequences can be deduced, the program needs to know the geometric constraints on assembly. While several researchers have attempted to obtain this information automatically from geometric data such as described above, such attempts rest on simplified geometry or assumptions that do not apply to real products. Much constraint information can be obtained directly from the logical data and the "direction" information about mating features, but inevitably some complex geometry must be analyzed or a multidimensional version of the piano mover's problem must be solved. Since the designer can usually solve such problems almost instantly if shown a sufficiently realistic drawing ${ }^{1}$ of the parts, we have chosen to use an illustrated question-answer method for obtaining any assembly constraint information that cannot be deduced logically from the features.[8] $\mathrm{A}$ typical moment in this method is shown in Figure 6 . The number of questions asked of the designer varies considerably from product to product; for the seeker 58 questions were asked. The computer answered 203754 others itself using feature information.

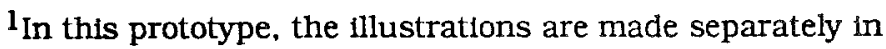
Sundraw ${ }^{T M}$.
} 

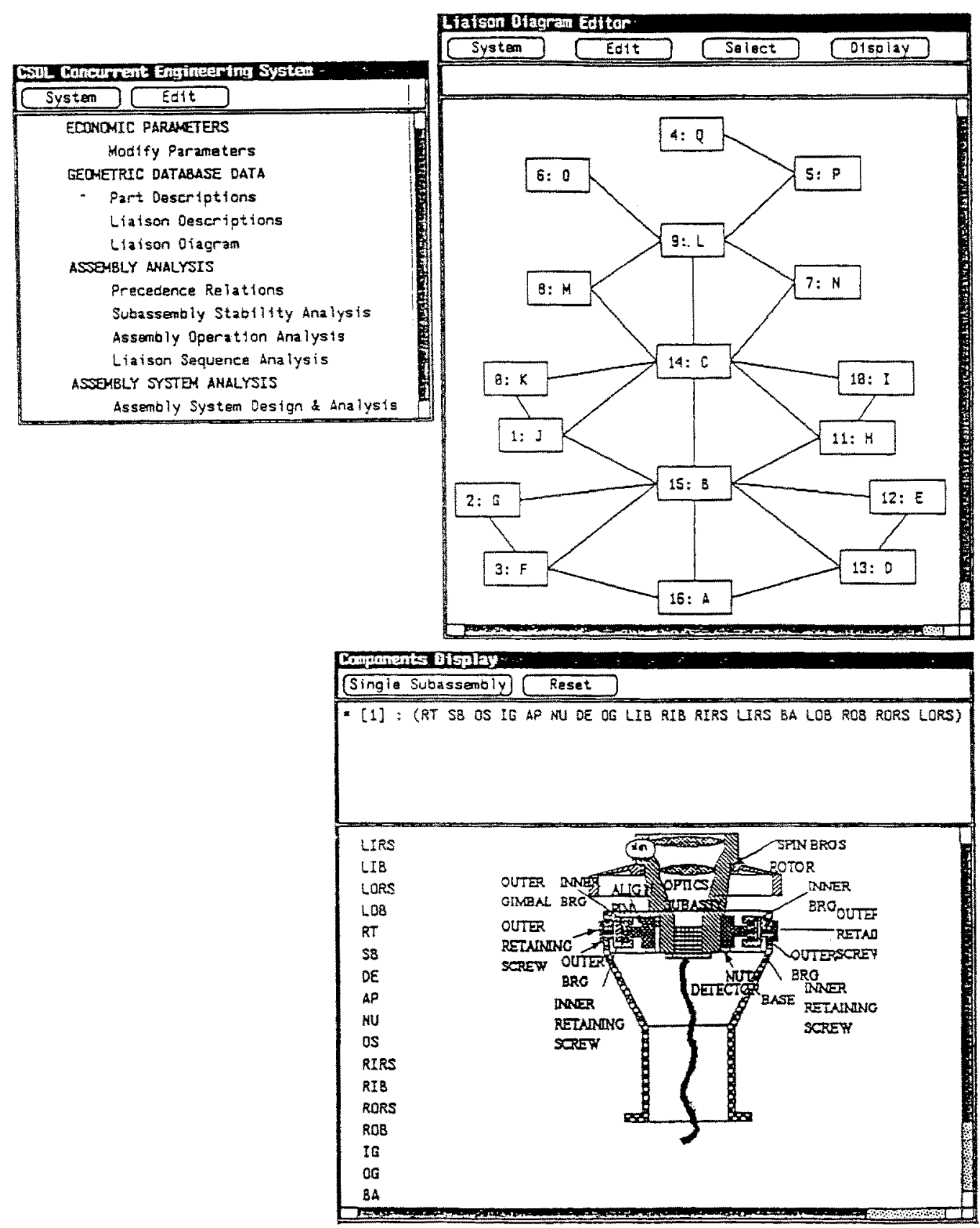

Figure 6. View of Screen During Question-Answer Session for Determining Assembly Constraints 


\section{Assembly Sequence Generator-Editor}

The assembly sequence generator uses the assembly constraints and creates a database and corresponding display (Figure 7) that maps out the feasible sequences. [10]

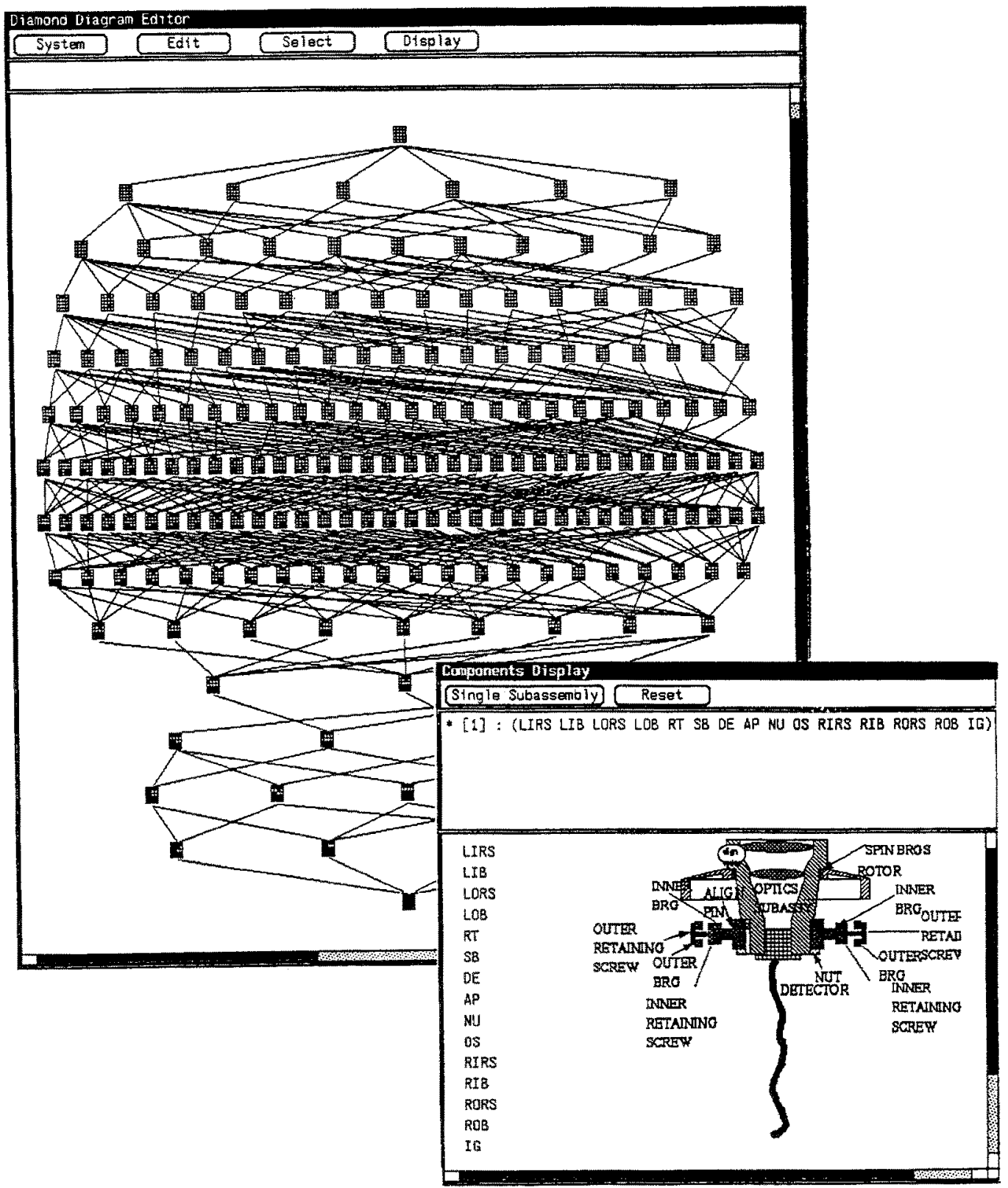

Figure 7a. View of the Screen Showing the Feasible Assembly Sequences for the Seeker After the Designer Has Edited Out Several Undesirable Assembly States and Moves. 


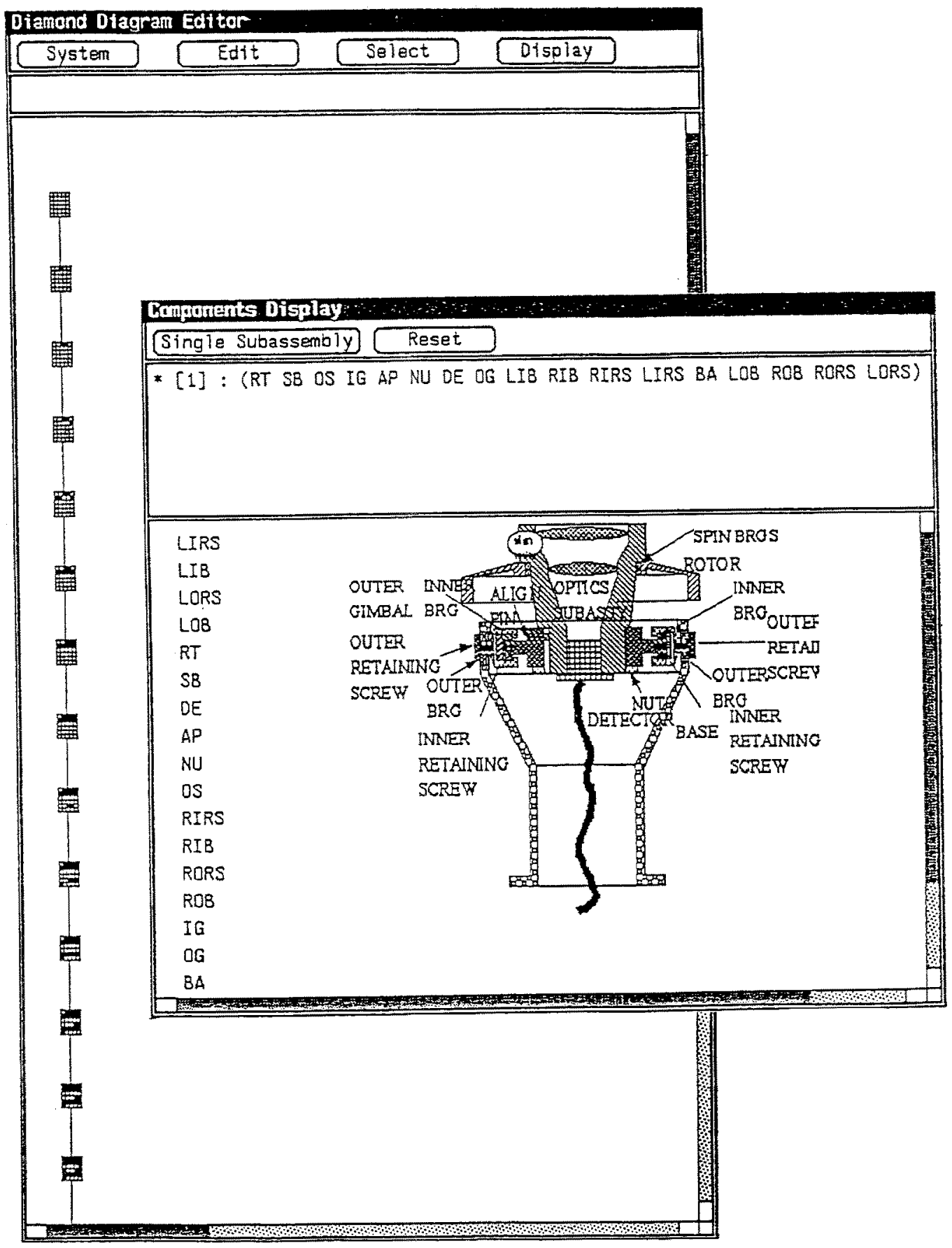

Figure 7b. View After Editing to One Sequence. Each box in the network is a state of assembly corresponding to mates that have been established. A path from top to bottom in this network is a unique assembly sequence. 
The user may click on any box and see a picture of the state of assembly it represents, or on any connected pair of boxes and visualize the assembly move it

represents. ${ }^{1}$ The user may delete any state or move, or remove all states containing more than one subassembly (to eliminate all but sequential single line assembly layouts), or write logical statements to eliminate undesirable situations such as simultaneous establishment of a specified group of mates. It is also possible to require that a mate or set of mates occur right after some other mate. By means of such edits the designer can consider the possibilities thoroughly and can arrive at a reasonable sequence in less than an hour for products similar to the seeker head in complexity and part count.

Not yet implemented but quite feasible are heuristic means for using feature information to accomplish preliminary editing of mates and subassemblies for the designer. In addition, a separate program [14] has implemented consideration of fixturing and orientation options, permitting the designer to choose a sequence with as few reorientations and refixturings as possible. This capability has not yet been merged with the prototype described here.

\section{Assembly Process Planner}

The assembly sequence(s) produced above consist of a series of mates in the desired order but they lack much other information necessary to a complete assembly plan. This information includes orientation, fixturing, application of adhesives, oven curing of adhesives, torquing of bolts, and so on. The data necessary to add this information can be determined from the geometric features or from information supplied by the designer. Figure 8 shows a plan for the gimbal portion of the seeker head.

${ }^{1}$ The Sundraw ${ }^{\mathrm{TM}}$ illustrations are used here, too. 


\begin{tabular}{|c|c|c|c|c|c|}
\hline \multicolumn{6}{|c|}{ System } \\
\hline Task & Type of Task & DaD & Mat Req & Load & Description \\
\hline 1 & OPlace & 3 & $\times Y \bar{g}$ & 8.2281 & Attach SPIN_BEARING-P to pallet. \\
\hline 2 & Assemble & $\overrightarrow{3}$ & $x Y \underline{Z}$ & 1.7788 & insert ROTOR-Q. \\
\hline 3 & S Automatic-reorient & 1 & 1 & 4.3297 & rotate pallet/trunion. \\
\hline 4 & OAssemble & 3 & $X Y \mathrm{~g}$ & 2.3318 & Insert OPTICS_SUBASSEM-L. \\
\hline 5 & SAutomatic-reortent & 1 & 10 & 4.7992 & rotate pallet/trunion. \\
\hline 8 & Splace & 1 & $\times 8$ & 0.3794 & Assemble INNER_GIMBAL-C. \\
\hline 7 & Autcmatic-reorient & 1 & 08 & 4.7899 & rotata pallet/trunion. \\
\hline 8 & X place & 1 & $x \mathrm{z}$ & $7.5 E-4$ & Assembia ALIGNMENT_FIN-N. \\
\hline 9 & SAutomatic-reorient & 1 & a & 4.8167 & rotate pallet/trunion. \\
\hline 18 & S8o1t & 2 & $X Y$ Y & 8.1867 & insert and torque RETAINING_NUT-M. \\
\hline 11 & SAutomatic-reorient & 1 & 즘 & 4.9439 & rotate pallat/trunion. \\
\hline 12 & Oplacs & 2 & . & 1 & Apply adhesives to surfaces. \\
\hline 13 & OInsert & 3 & $X Y \mathrm{~g}$ & 9.1272 & Drient and install DETECTOR-O. \\
\hline 14 & Oplace & 1 & $\times \bar{Z}$ & 4.9439 & Dven cura. \\
\hline 15 & Oplaca & 2 & $\times Y \bar{\Omega}$ & 1 & Position and attach fixture to palle \\
\hline 16 & Splace & 1 & $\times \mathbb{2}$ & 8.6184 & Assemble OUTER_GIMBAL-B. \\
\hline 17 & Qutomatic-reorient & 1 & . & 5.5693 & rotate pallet/trunion. \\
\hline 18 & Ossemble & 3 & $X Y$ Z & 9.8149 & insert R-2_BEARING-J. \\
\hline 19 & $\approx$ Automatic-reorient & 1 & 1 0 & 5.5842 & rotate pallet/trunion. \\
\hline 28 & OAssemble & 3 & $x Y$ 马 & 8.0149 & Insart R-2_BEARING-H. \\
\hline 21 & Automatic-reorient & 1 & 可 & 5.5983 & rotate pallet/trunion. \\
\hline 22 & Osolt & 2 & $X Y \boxminus$ & 0.8068 & insert and torque SCREY_2-64-I. \\
\hline 23 & Automatic-reorient & 1 & D & 5.5963 & rotata pallat/trunion. \\
\hline 24 & SBolt & 2 & $X Y E$ & 0.8868 & Insert and torque SCREY_2-64-K. \\
\hline 25 & OPlace & 2 & $X Y \mathrm{~g}$ & 4 & Position and attach fixture to pall \\
\hline 26 & Oplace & 1 & 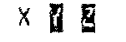 & 3.8978 & Assemble BASE-A. \\
\hline 27 & $\approx$ Automatic-reortent & 1 & 78 & 9.5198 & rotate pallet/trunion. \\
\hline 28 & Cassembia & 3 & $X Y \Omega$ & 0.8256 & insert R-3_BEARING-F. \\
\hline 29 & $\mathcal{O}$ Automatic-reortent & 1 & 1) & 9.5455 & rotate pallet/trunion. \\
\hline 38 & Oassemble & 3 & $X Y \underline{z}$ & 8.3256 & insert R-3_BEARING-D. \\
\hline 31 & SAutomatic-reorient & 1 & 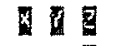 & 9.5528 & rotate pallet/trunion. \\
\hline 32 & Solt & 2 & $X Y \underline{Z}$ & 8.8873 & insert and torque SCREY_ $4-48-E$. \\
\hline 33 & S Automatic-reorient & 1 & ] & 9.5681 & rotate pallet/trunion. \\
\hline 34 & S801t & 2 & $X Y Z$ & 8.8873 & insert and torque SCREY_4-48-6. \\
\hline 35 & SAutomatic-test & 2 & DE & 8.8 & perform test \\
\hline 36 & Oplace & 1 & ] & 9.5681 & pack / unioad assembly. \\
\hline
\end{tabular}

Figure 8. Assembly PIan for the Seeker's Gimbal 
For example, if a ball bearing is to be installed and there is no bearing retainer, then it will be installed with a shrink or interference fit, or glued in, either with epoxy or Loctite $^{T M}$. In the case of epoxy, an oven cure is necessary. In the case of bolt torquing, the size of the bolt usually determines the necessary torque directly. For fixturing, the feature information contains the approach direction for each mate. If we adopt the heuristic that mates should be made from above if possible, then the need for reorientation can be deduced. We also assume that the first part in a sequence must be placed on a fixture. Also, each separate subassembly built needs its own fixture.

Finally, it may happen that a part must be added to an assembly but not immediately mated to the other parts; instead it must be put on a separate temporary fixture. The need for such a temporary fixture cannot be deduced directly from the assembly sequence method described above because it presumes that each assembly step establishes at least one mate. However, it sometimes happens that interferences between parts require that parts be placed near each other, followed by the insertion of intervening mating parts; if the intervening parts were added first, then one of the other parts could not be inserted. This situation is illustrated in Figure 9.

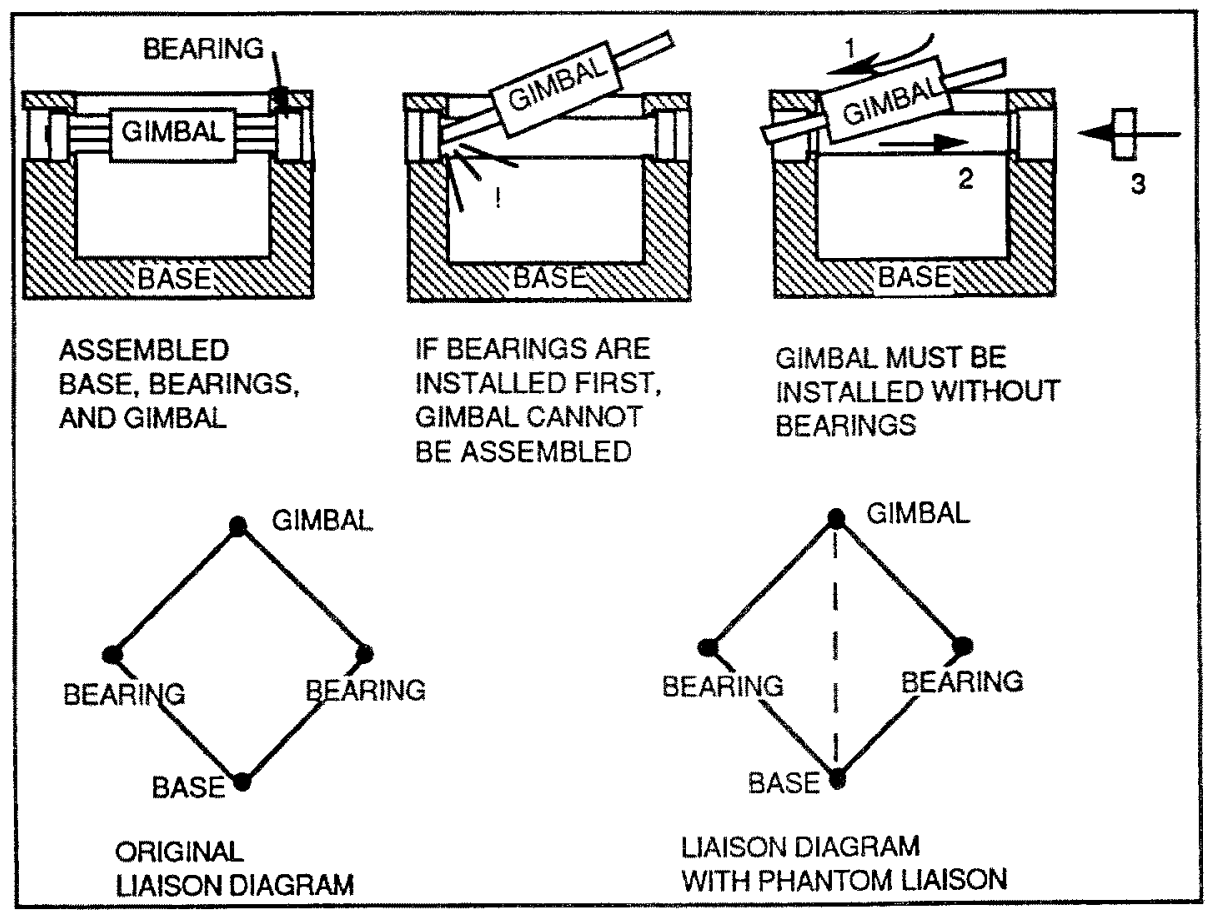

Figure 9. Assembly of Parts That Do Not Mate Directly, Showing Use of the Phantom Liaison. 
At present the designer must supply this information by adding a "phantom liaison" between the two main parts. Since establishing this liaison does not mate any parts, the temporary fixture is called for. If the phantom liaison were omitted, there would be no feasible assembly sequences.

When the software has finished making the process plan, the designer may review it and make any changes desired.

\section{Assembly System Designer}

The last step accomplished by the prototype design software is assembly system design. This step converts the assembly process plan into a list of equipment and tools together with the plan steps that each equipment will accomplish. Equipment includes manual assembly and maintenance labor, robots, and conventional fixed assembly automation. The final cost of assembly is also calculated, based on labor and equipment costs. Some non-feature information is required to provide economic and production data, such as cost of labor, desired annual production quantity, and information on investment return requirements.

Process planning is carried out heuristically using feature information such as bounding box size of subassemblies, weight of parts, and type of mate. Larger or heavier parts, or longer reach required of assembly equipment (calculated from the size of the assembly's bounding box) require more costly equipment, more assembly time, or both. Heuristics determine task difficulty based on type of mate, sizes and weights of parts, and number of features that mate simultaneously. The task difficulty score is used to rule out certain types of equipment and to determine equipment cost estimates.

The algorithm seeks to obtain the lowest unit cost of assembly, and uses equipment costs and speeds to decide which equipment to choose for each step. "Flexible" equipment such as manual labor or robots can do more than one task so the algorithm can assign several tasks to one such piece of equipment if time in the assembly cycle is available. See $[2,9]$ for more information on how these calculations are made.

The resulting plan is presented to the designer, who can alter it as desired. Figure 10 shows the system design for the gimbal portion of the seeker. 

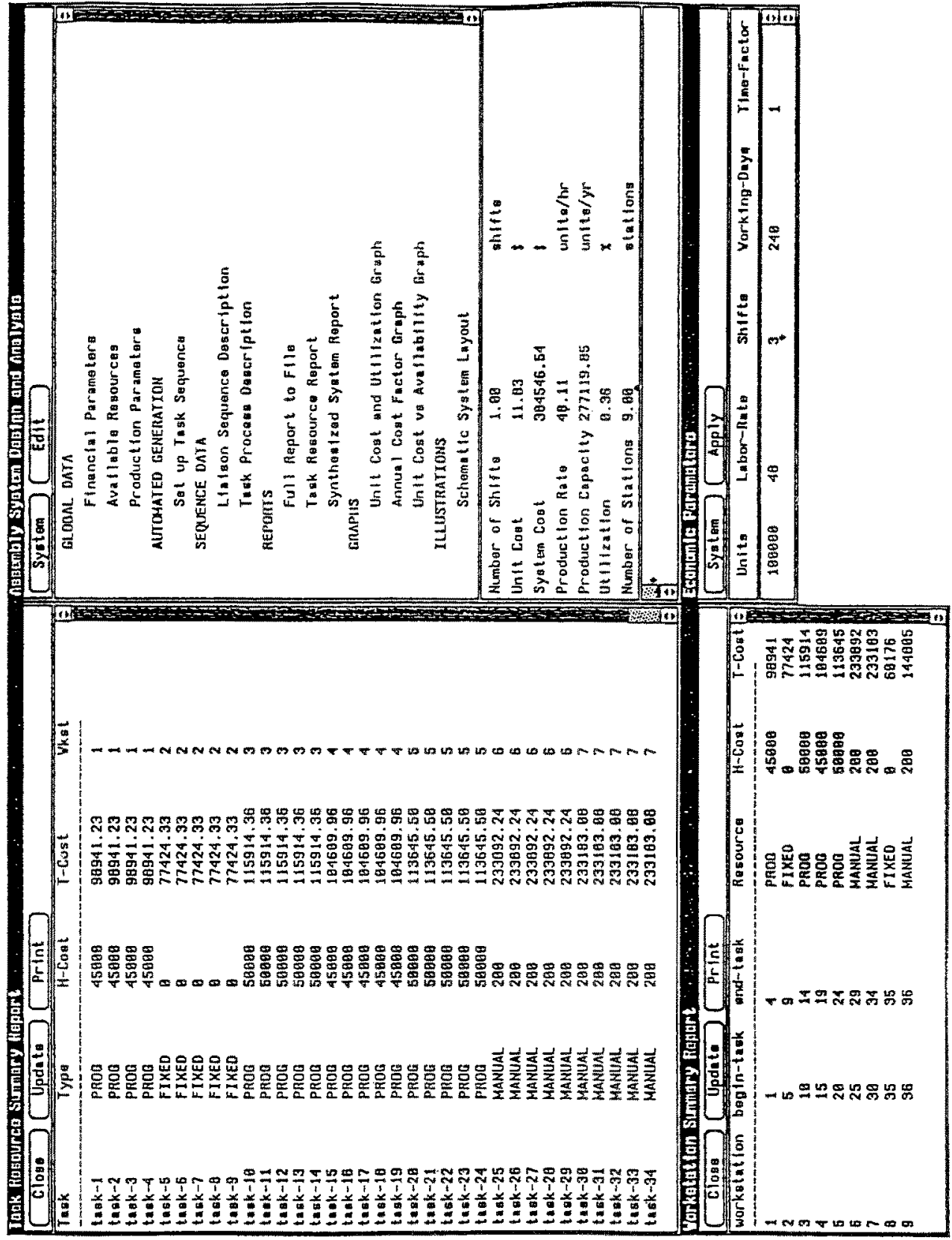

Figure 10. Assembly System Design Data for the Seeker Gimbal 


\section{CONCLUSIONS AND FUTURE WORK POSSIBLE WITHIN THIS ARCHITECTURE}

The information needs of the design for assembly analyses we have investigated which can be provided through feature-based modeling are;

1. Topology of the assembly, connections between parts must be explicit and should be described as feature to feature mates. This also makes assembly modeling easier by allowing the user to position parts just by specifying the mates.

2. Interface features, knowing that a mate is composed of a spline in a through hole, as opposed to a peg in a blind hole, is important to subsequent assembly analyses.

3. Links from features to geometric database, having access to the geometry that represents a part or features, provides DFA analyses the power of both a symbolic and quantized representation.

4. Feature parameters, the parameters used to create the features, such as diameter of a hole must be stored with the feature. This allows subsequent calculation of clearances and assembly difficulty without having to parse the geometric database.

5. Tolerances on feature parameters and part dimensions will become extremely important to the more detailed DFA analyses described later.

It should be noted that a properly designed feature-based modeling system can both, meet the information needs of the downstream analyses, and yield a significant improvement in productivity of geometric modeling.

We now have the basis for feature-based design for assembly. It consists of a means for describing parts and assemblies together with the important features that describe assembly operations, plus algorithms and displays that permit a designer to explore the assembly opportunities, discover the assembly problems inherent in the design, and correct them at an early stage. Many aspects of design are not included but the basis for doing so exists. These are briefly discussed next.

\section{Incorporation of Part-Based DFA}

A wealth of information exists concerning ability to feed and insert individual parts. [11] Most of this information can be expressed in terms of part features. It should be fairly straight-forward to combine this information with the software system described here.

\section{Critique of Part Mate Difficulty}

The science of part mating is well-established and is described in reference [2]. This theory permits direct assessment of the difficulty of part mates on the basis of relative clearance, size of chamfers (if any), friction, compliance of grippers and fixtures, and accumulated position and angle errors between mating parts at the moment of assembly. A first stage assessment of difficulty can be obtained simply from the clearance, chamfer, and friction information, which in turn is found directly in the feature information. For example, 
a relative clearance (clearance divided by diameter) in a round peg-hole mate of less than 0.001 and friction coefficient of more than 0.2 may be judged difficult.

To complete the picture of assembly difficulty requires additional calculations involving tolerance stackup in assemblies, fixtures, and assembly equipment. One needs to know what surface on what part is being used for fixturing, plus tolerances on all the intervening parts across the features being used for mating. One also needs to know something about the accuracy and repeatability of assembly equipment. Alternatively, one can calculate the requirements on such equipment to obtain, say, "six sigma" performance. The information for making such calculations exists within the features and the assembly plan. However, due to the undeveloped state of tolerance representation in geometric modeling, additional research is needed before the addition of such calculations will be straightforward. [12]

\section{Critique of Part Cost}

As each part is designed, the designer should be given information on its fabrication cost. Such information depends on feature information, especially shapes and tolerances, as well as choices by the designer concerning fabrication methods and materials. This is a separate and active topic of research and development. There is no reason, however, why the results of such research cannot be incorporated into the system described here. [6,7]

\section{Critique of Assembly Automation Opportunities}

The heuristic assembly system design algorithm makes numerous choices of equipment based on mating information. Such choices are not presently communicated to the designer. It would be straightforward to do so, however, permitting the designer to explore design changes that might permit more options for equipment and possibly yield lower assembly costs.

\section{Analysis of Fixturing Requirements}

At present the software makes only preliminary fixturing analyses. Additional opportunities, ranging from straightforward to challenging, include reorientations, fixture interference with parts, clamping, and tolerance stackup.

\section{Inclusion of Testing Requirements}

One may conclude from the above discussions that assembly includes much more than just putting parts together. An important element not mentioned above is testing, in particular in-process testing of partially completed assemblies. In-process testing may be advantageous because problems can be found sooner and more easily, and can be repaired more easily compared to testing only after assembly is complete.

However, there are several complications. First, some possible failures can be detected only at final test. Second, in-process tests require the presence of certain parts and 
completion of certain assembly steps. Third, tests at some points during assembly may be able to detect failure but be unable to determine the cause, requiring diagnosis. Consequently, planning of in-process testing depends on the choice of assembly sequence. In separate research [13] we have begun to work on the problem of determining optimum testing opportunities. Full exploitation of this aspect of assembly requires statistical data concerning likelihood of certain failures, plus the costs of test equipment, cost and time to make repairs, and information about the interaction of test capabilities and failures present.

\section{References}

1. Anon, "Requirements for Support of Form Features in a Solid Modeling System," Final Report R-85-ASPP-01, CAM-i, Inc., Arlington, TX, 19851.

2. J. L. Nevins, D. E. Whitney, editors, Concurrent Design of Products and Processes, New York: McGraw-Hill, 1989.

3. E. Kroll, E. Lenz, J. R. Wolberg, "A Knowledge-based Solution to the Design for Assembly Problem," Manufacturing Review, v1, \#2, June, 1988, pp104-108.

4. M. R. Cutkosky, J. M. Tenenbaum, "CAD/CAM Integration Through Concurrent Process and Product Design," ASME Symposium on Integrated and Intelligent Design, 1987.

5. R. D. Logcher and D. Sriram, "CAE Techniques for Distributed Design," to be presented at Int'1 Symposium on Building Economics and Construction Management, Sydney Australia, March, 1990.

6. S. C. Luby, J. R. Dixon, and M. K. Simmons, "Designing with Features: Creating and Using a Features Database for Evaluation of Manufacturability of Castings". Proceedings of the International Computers in Mechanical Engineering Conference of the American Society of Mechanical Engineers, Chicago IL, August 1986. Reprinted in CIME Magazine, Vol.5, No. 3, November, 1986 pp. 25-33.

7. P. London, B. G. Hankins, S. C. Luby, and M. Sapposnek, "The Expert Cost and Manufacturability Guide: A customizable Expert System", Proceedings of the International Computers in Mechanical Engineering Conference of the American Society of Mechanical Engineers, New York, NY, August 1987.

8. D. F. Baldwin, T. E. Abell, M-C M. Lui, T. L. De Fazio, D. E. Whitney, "An Integrated Computer Aid for Generating and Evaluating Assembly Sequences for Mechanical Parts," submitted to IEEE J. Robotics and Automation.

9. R. E. Gustavson, "Design of Cost-Effective Assembly Systems," SME Paper AD88250, presented at Successful Planning and Implementation of Flexible Assembly Systems, March 29-31, 1988, Ann Arbor, MI.

10. M-C M. Lui, "Generation and Evaluation of Mechanical Assembly Sequences Using the Liaison Sequence Method," MIT SM Thesis, ME Department, May, 1988.

11. G. Boothroyd and P. Dewhurst, Product Design for Assembly, Wakefield RI: Boothroyd and Dewhurst, Inc., 1987 
12. J. Turner, "An M-Space Theory of Tolerances in Solid Modeling," proceedings NSF Design and Manufacturing Systems Grantees Conference, Tempe AZ, January, 1990, published by SME.

13. S. Pappu, "A Dual Descent Algorithm for Finding the Optimal Test Strategy for an Assembly Sequence, MIT SM Thesis, Operations Research Center, May, 1989.

14. T. E. Abell, "An Interactive Tool for Editing and Evaluating Mechanical Assembly Sequences Based on Fixturing and Orientation Requirements, "MIT SM Thesis, ME Department, 1989. 